\title{
La justificación de la agresión física entre políticos colombianos. El caso del exalcalde Rodolfo Hernández en YouTube
}

\section{Giohanny Olave ${ }^{1}$}

Recibido: 23/01/2020

Aprobado por pares: 01/03/2020
Enviado a pares: $24 / 01 / 2020$

Aceptado: 02/04/2020

DOI: 10.5294/pacla.2021.24.4.2

Para citar este artículo / to reference this article / para citar este artigo Olave, G. (2021). La justificación de la agresión física entre políticos colombianos. El caso del exalcalde Rodolfo Hernández en YouTube. Palabra Clave, 24(4), e2442. https://doi. org/10.5294/pacla.2021.24.4.2

\section{Resumen}

En noviembre de 2018, el entonces alcalde de Bucaramanga (Colombia) agredió físicamente a un concejal del municipio, cuando mantenían una discusión política acalorada. El video de la agresión circuló profusamente en redes sociales y fue utilizado por la Procuraduría General de la Nación como prueba fehaciente para suspender del cargo al alcalde. En este artículo se analiza un conjunto de comentarios virtuales de internautas en reacción al video de las agresiones. El corpus de comentarios se extrajo de la publicación realizada por la casa de noticias El Tiempo en la red social YouTube, a través de la herramienta YouTube Comment Scraper, entre el 28 de noviembre y el 28 de diciembre de 2018, para concentrarse en las reacciones que el hecho político suscitó durante el primer mes en que fue publicado. Desde una perspectiva interpretativa interdisciplinar del Análisis del

$1 \triangle$ https://orcid.org/0000-0001-6794-6472. Universidad Industrial de Santander, Colombia. giolavar@uis.edu.co 
Discurso se determinan las regularidades de esas reacciones, los modos de justificar explícita e implícitamente los actos violentos y los efectos políticos que terminaron por favorecer la imagen pública del alcalde en ese momento. Se concluye insertando el caso estudiado dentro de las discusiones actuales sobre la representación democrática y el ascenso de matrices ideológicas antipolíticas en el discurso público de quienes gobiernan.

\section{Palabras clave (Fuente: tesauro de la Unesco)}

Agresión; comunicación interactiva; comunicación política; comportamiento animal; discurso; etología; liderazgo político; opinión pública; violencia. 


\section{Justifying Assault between Colombian Politicians. The Case of Former Mayor Rodolfo Hernández on YouTube}

\section{Abstract}

In November 2018, the then-mayor of Bucaramanga (Colombia) attacked a municipal councilor amidst a heated political discussion. The video of the assault spread like wildfire on social media and was used by the Attorney General's Office as reliable evidence to suspend the mayor from office. This article analyzes a set of virtual comments from Internet users in reaction to the video. The corpus was extracted from the post made by the $E l$ Tiempo news outlet on YouTube using the YouTube Comment Scraper tool between November 28 and December 28, 2018, to focus on reactions to the political event in the first month. From an interdisciplinary interpretive perspective of discourse analysis, we determined the regularities of these reactions, the ways of explicitly and implicitly justifying acts of violence, and the political effects that ended up favoring the former mayor's public image. The article concludes by inserting the studied case into the current debates on democratic representation and the rise of anti-political ideological matrices in rulers' public discourse.

\section{Keywords (Source: Unesco Thesaurus)}

Aggression; interactive communication; political communication; animal behaviour; speech; ethology; political leadership; public opinion; violence. 


\section{A justificativa da agressão física entre políticos colombianos. 0 caso do ex- prefeito Rodolfo Hernández no YouTube}

\section{Resumo}

Em novembro de 2018, o então prefeito de Bucaramanga (Colômbia) agrediu fisicamente um vereador, quando mantinham uma discussão política acalorada. $\mathrm{O}$ vídeo da agressão circulou profusamente nas redes sociais e foi utilizado pela Procuradoria-geral da Nação como prova fidedigna para suspender o prefeito do cargo. Neste artigo, é analisado um conjunto de comentários virtuais de internautas em reação ao vídeo das agressões. $\mathrm{O}$ corpus de comentários se retirou da publicação realizada pela casa de notícias El Tiempo na rede social YouTube, por meio da ferramenta YouTube Comment Scraper, entre 28 de novembro e 28 de dezembro de 2018, para concentrar-se nas reações que o fato político suscitou durante o primeiro mês em que foi publicado. De uma perspectiva interpretativa interdisciplinar da análise do discurso, são determinados as regularidades dessas reações, os modos de justificar explícita e implicitamente os atos violentos e os efeitos políticos que acabaram favorecendo a imagem pública do prefeito nesse momento. Conclui-se com a introdução do caso estudado dentro das discussões atuais sobre a representação democrática e a ascensão de matrizes ideológicas antipolíticas no discurso público de quem governa.

\section{Plavras-chave (Fonte: tesauro da Unesco)}

Agressão; comunicação interativa; comunicação política; comportamento animal; fala; etologia; liderança política; opinião pública; violência. 
En noviembre de 2018, el entonces alcalde de la ciudad de Bucaramanga ${ }^{2}$ agredió físicamente a un concejal del municipio, cuando mantenían una discusión política acalorada. El video de la agresión circuló profusamente en redes sociales y fue utilizado por la Procuraduría General de la Nación como prueba fehaciente para suspender termporalmente al alcalde, quien había sido electo para un periodo de cuatro años, desde enero de 2016. En este artículo se analiza un conjunto de comentarios virtuales de internautas en reacción con el video de las agresiones. El objetivo es determinar las regularidades de esas reacciones, analizar los modos de justificar explícita e implícitamente los actos violentos y los efectos políticos que terminaron por favorecer la imagen pública del alcalde en ese momento. Se propone un acercamiento discursivo a las reacciones y modos como las expresan los internautas al apoyar o sancionar la conducta agresiva de un líder político. Dado que el análisis es discursivo, se orienta hacia el ejercicio interpretativo de una situación comunicativa presentada como una práctica social, históricamente situada y analizada desde un enfoque interdisciplinar y crítico (Arnoux, 2019).

En particular, en la interpretación ofrecida interesa involucrar la mirada explicativa sobre las agresiones físicas, provista por los estudios etológicos (Eibl-Eibesfeldt, 2017; Hammerstein, 2013; Kortüm y Heinze, 2013; Schmitt et al., 1997). La aproximación etológica a los intercambios discursivos ya fue utilizada por Fracchiolla (2013, p. 35) y por Olave (2019) para preguntarse por las causas que hacen aparecer agresiones verbales en el espacio público, más allá de la pregunta por sus finalidades o por las intenciones de los sujetos al usarlas. En ese marco, la agresión es concebida como un instinto o disposición filogenética con funciones sociales y anclajes intra e interespecíficos -sujeta, claro está, a demandas ambientales y a modificaciones adaptativas mediante experiencias de aprendizaje-, más que como una deformación de las interacciones políticas, cuya supresión dependería solo de la voluntad racional de los sujetos o de las normas sociales que regulan sus conductas:

2 Municipio colombiano ubicado al nororiente del país, capital del departamento de Santander. 
Podemos definir como agresivos aquellos comportamientos a través de los cuales el hombre y los animales protegen sus intereses contra la resistencia de otras especies, y de este modo tratan de mantener dominio sobre ellas [...]. El comportamiento agonal está jerárquicamente organizado y comprende subsistemas de lucha (conducta agresiva y defensiva) y escape (elusión y sumisión). (Eibl-Eibesfeldt, 2017, p. 364)

Desde el punto de vista etológico, entonces, la agresión humana tiene una relación directa con la defensa o protección de los intereses del individuo cuando se perciben amenazados. Una parte importante de la defensa de esos intereses tiene que ver con la demarcación de los grupos identitarios o "endogrupos" en las dinámicas de organización social (Eibl-Eibesfeldt, 2017, p. 296), específicamente, la protección de la estructura familiar y del territorio de asentamiento.

En este texto hablaremos de defensa del endogrupo familiar en ese sentido etológico, para sustentar la interpretación de las reacciones frente a la agresión física. Sin embargo, como se trata de un análisis de discurso y no de un estudio etológico, los conceptos no serán aplicados directamente, sino que serán utilizados para movilizar las interpretaciones de los comentarios recolectados. Dentro del análisis discursivo, la visión etológica puede ayudar a explicar las reacciones que construyen tomas de postura frente a interacciones hostiles en las cuales los debates se convierten en combates, como en el caso que motiva este estudio.

\section{Antecedentes}

Desde su aparición en la escena política colombiana (a partir de su campaña a la alcaldía en 2015), el exfuncionario Rodolfo Hernández construyó una imagen pública cimentada en la independencia partidista y en una agenda ética contra la corrupción gubernamental. Para Briceño et al. (2017, p. 54), se trata de un "actor político emergente" que logró capitalizar el descontento con la clase política tradicional en la región y el malestar democrático general, a partir de la figura del outsider político (Novaro, 2000), en este caso, proveniente del ámbito ingenieril y empresarial. 
Las plataformas digitales son utilizadas frecuentemente en la comunicación política contemporánea e incluyen el uso intensivo de redes sociales por parte de los funcionarios y aspirantes a cargos públicos (Lanza y Fidel, 2011). En este escenario de la política, Briceño et al. (2018) mostraron que la figura de Rodolfo Hernández como outsider logró favorabilidad entre los internautas de la plataforma Facebook y capitalizar la "desafección política” (Abad y Track, 2013; Di Palma, 1970; Salcedo y García, 2015) de la ciudadanía durante la campaña a la alcaldía en 2015.

Los trabajos referidos de Briceño et al. (2017, 2018), a propósito de la recepción de la figura de Rodolfo Hernández en Facebook, proponen que el registro en las redes sociales es una vía para el diagnóstico -siempre parcial- de la cultura política (Almond, 1990) y de fenómenos como el de la desafección institucional y la indiferencia frente a los partidos. Este artículo extiende ese presupuesto a los foros virtuales o "ciberforos" en la plataforma YouTube, en los cuales los internautas registran comentarios a los videos publicados.

Los comentarios digitales en estas plataformas han sido considerados como formas de participación política y cultural en las "videocracias" contemporáneas (Alloca, 2018; Burgess y Green, 2009), frente a temas que habilitan controversias y opiniones diversas. Desde otros puntos de vita, en contraste, YouTubereproduce la banalidad de la sociedad del espectáculo y de las industrias culturales capitalistas (Bañuelos, 2009), participa en la producción de estereotipos sociales y discursos hegemónicos (Pardo, 2012) y replantea la interacción entre los sujetos empíricos, ahora como estructuras simbólicas que interactúan en clave de simulacro y conflicto (Scolari, 2004). De cualquier forma, según Schneebeli (2015), a través del comentario en YouTube el internauta se ubica en un lugar social y en una escala afectiva, epistémica y social; asimismo, se posiciona y relaciona a los interlocutores virtuales entre sí y a los temas que comenta.

Existe un buen número de aproximaciones a esa "toma de postura" o stance-taking en la bibliografía contemporánea disponible (Barton y Lee, 2013; Englebretson, 2007; Jaffe, 2009). Centrados en la red YouTube, se 
destaca la clasificación que ofrece Schneebeli (2015), basado en Du Bois (2007), de las modalidades lingüísticas que los usuarios de YouTube utilizan para tomar posición en sus comentarios: evaluación, autoposicionamiento, alineamiento e invectiva. Con un mayor énfasis en las percepciones de los internautas, Lange (2014) encuentra que frente a videos de diatribas ("YouTube rants") se generan reacciones emocionales solidarias que desplazan la crítica de las formas expresivas agresivas por la discusión acerca de los objetos o temas evaluados negativamente. En el mismo sentido, Kwon y Gruzd (2017) demuestran que la agresión es imitada por los usuarios en línea, especialmente el acto de maldecir en YouTube al comentar e interactuar en torno a videos de campaña electoral.

Por su parte, Moor et al. (2010) interrogan directamente a los internautas acerca de sus percepciones sobre las discusiones acaloradas en YouTube (flaming) y concluyen que son sancionadas en general por transgredir las normas comunicativas y empáticas en la red. Un trabajo reciente de Graf et al. confirma esa percepción negativa de los internautas frente a los comentarios virtuales descorteses, inclusive si aparecen o no anonimizados. Para Graf et al. (2017, p. 543), el incremento del uso de estos foros virtuales en las noticias en línea ha puesto de relieve la necesidad de regular esa práctica discursiva por parte de los medios que ofrecen la posibilidad de hacer comentarios, de acuerdo con el principio de la promoción de un registro cortés o "civilizado". Esta aspiración, sin embargo, puede verse influida por el tipo de contenido que se comenta: el material polémico y político es una fuente de comentarios interesante para revisar el comportamiento verbal de los internautas, pues podría influir en el grado de civilidad y en el desarrollo de las discusiones en los comentarios en línea (p. 544). La explicación que ofrecen Ksiazek et al. (2015, p. 864) para la civilidad y su contraparte, la hostilidad, en los comentarios virtuales se orienta en el mismo sentido: videos de noticias más populares y comprometidas con puntos de vista tienden a generar discusiones más hostiles, lo cual disminuye el potencial deliberativo de la interacción en línea. McCosker (2013) destaca que el perfil de esas discusiones en YouTube constituye una forma de agonismo público que requiere interpretaciones contextuales, pues las provocaciones y demás formas responsivas hostiles pueden ser vistas también 
como formas de participación política dentro de lo que Mouffe (2003) denomina "pluralismo agonista". Este mismo modelo de democracia "radical" es la base teórica que sustenta las reflexiones de Amossy (2014) acerca de la polémica pública como un modo de "coexistencia en el disenso", esto es, de verbalizar los desacuerdos para impedir la violencia física.

De otro lado, en un trabajo de rastreo de tipos de generadores de contenido y de audiencia, May (2010) demuestra cuantitativamente que en la red social YouTube los sitios de noticias y política se ven especialmente impulsados por poderosos medios corporativos (agencias de noticias, como AP o ABC News), con construcción de grandes audiencias. Desde ese punto de vista de la producción de contenidos, Berry y Sobieraj (2014) hablan de la producción y orientación de emociones en el ámbito público, siempre atravesadas por intereses económicos que afectan el desarrollo de discusiones políticas enriquecedoras y afectan el funcionamiento de las instituciones democráticas. Los autores se refieren, específicamente, a lo que denominan "industria de la indignación", pero su trabajo puede extenderse a la producción de emociones públicas en internet.

Finalmente, esta última perspectiva centrada en la conducción política de las emociones públicas (Staiger et al., 2010) o "regímenes emocionales" (Reddy, 2001) ha dado lugar a una multiplicidad de trabajos ensayísticos y de investigación acerca del discurso de Donald Trump, en el ámbito estadounidense. Para Morini (2020), el caso Trump es ejemplar para entender el papel crucial de las emociones y de la "retórica agresiva" en la relación entre el líder político y su audiencia, vínculo afectivo que termina desplazando los contenidos y las discusiones democráticas de fondo. Morini (p. 88) postula que el lenguaje populista y violento es efectivo porque es percibido como cercano, transparente, simple e informal por parte de los ciudadanos. Wahl-Jorgensen (2019, p. 79) se refiere al mismo fenómeno como un régimen emocional de la ira indiscriminada, que conduce o promueve la demostración pública del descontento. Las implicaciones de ese régimen problematizan el papel actual de los medios informativos, cuya crisis financiera dentro de las lógicas de internet ha hecho que orienten las noticias hacia el infoentretenimiento (Happer et al., 2019; Boczkowski y Papacharissi, 2018). 


\section{Metodología}

El propósito de este avance de investigación es identificar regularidades discursivas en las reacciones de internautas que comentaron el video de la agresión física del exalcalde Hernández, difundido a través de YouTube. Se parte de la hipótesis principal según la cual ese tipo de comentarios digitales frente a la agresión física en escenas políticas da cuenta de emociones básicas compartidas que llevan a justificar esas conductas, aun tratándose de comportamientos directamente violentos. Así, las reacciones de los internautas ponen en escena un conjunto de emociones ciudadanas y de "tomas de postura" en el ámbito de la política local, mediada por las grandes empresas en internet que orientan la noticia como espectáculo o entretenimiento.

Se analizaron los comentarios realizados por internautas al video de la agresión publicado en el canal oficial de El Tiempo en YouTube, canal que cuenta con 445.000 suscriptores, la mayor cantidad de visualizaciones, en comparación con otros canales que también lo publicaron (en total, con 896.180 vistas) y que el medio periodístico publicó sin ediciones visibles ni narración periodística (excepto la identificación del canal al video superpuesta al original). La pieza audiovisual tiene una duración de 2:32 minutos y fue titulada "Alcalde de Bucaramanga golpea a concejal". El video fue valorado positivamente por los internautas, como lo evidencia la relación de 7.844 "me gusta" frente a 470 "no me gusta”.

A través de la herramienta YouTube Comment Scraper (Klostermann, 2015) se descargó una matriz de datos con todos los comentarios (2.066) que los internautas publicaron en el canal de YouTube de El Tiempo, desde la aparición del video hasta el 30 de septiembre de 2019. Para el análisis, se seleccionaron todos los comentarios realizados entre el 28 de noviembre y el 28 de diciembre de 2018, para concentrarse en las reacciones que el hecho político suscitó durante el primer mes en que fue publicado. Los ejemplos que apoyan la argumentación en este artículo fueron transliterados respetando su tipografía y ortografía; cuando se añaden elementos para destacar algunos aspectos, se indica al finalizar la cita. 
El corpus de comentarios digitales está conformado por un total de 488 intervenciones de los internautas, pero se omiten sus interacciones. Se trata de una muestra acotada, que no aspira a la generalización de conclusiones, sino al estudio detenido del caso focalizado, dada su singularidad en el escenario político (una agresión física directa de un alcalde, captada en cámara y difundida ampliamente en una red social); por tanto, no se realiza un análisis de big data ni una etnografía virtual (Dyke, 2013), sino el estudio de un caso particular significativo (Eisenhardt, 1989; Forni, 2011; Stake, 1995; Yin, 1984), elegido de manera intencional, en razón de sus características atípicas o extremas (Forni, 2011, p. 67).

El método corresponde al análisis del discurso en perspectiva interpretativa e interdisciplinar (Arnoux, 2019), según el cual se exploran interpretativamente materiales textuales en busca de regularidades y de hipótesis que desmonten y expliquen los mecanismos internos que construyen y orientan sentidos. El procedimiento es inductivo, producto de una codificación abierta y de una posterior codificación axial (Strauss y Corbin, 2002). En el software Numbers 6.1 se extrajeron las categorías emergentes, sustentadas en dos criterios principales: reacciones verbales frente a la conducta del alcalde y expresiones sobre la situación mostrada en el video. El enfoque del análisis es mixto (Hernández et al., 2017, p. 10), de manera que la integración y discusión conjunta de datos cuantitativos y cualitativos permitiera aproximarse a una interpretación más comprensiva del fenómeno. Así, la categorización fue procesada frecuencialmente para determinar las regularidades en términos porcentuales y focalizar el análisis cualitativo en las de mayor predominancia y significado en su dimensión política.

Las categorías emergentes no son discretas, sino indicativas de acentos u orientaciones de la toma de postura en los comentarios digitales capturados. Por esta razón, no se propone como resultado una taxonomía, sino una organización de los sentidos emergentes en los comentarios digitales a través de la interpretación de sus recurrencias y énfasis, con posibles solapamientos entre los rasgos que definen a cada grupo de comentarios delimitado. La argumentación de las categorías emergentes tiene en cuenta este criterio no clasificatorio y se orienta hacia la interpretación de los sentidos políticos de las reacciones al video por parte de los internautas. 
Es necesario aclarar que este artículo presenta el primer avance de una investigación mayor dentro de la cual se inserta; por lo tanto, los resultados no son finales, sino que constituyen un punto de partida para las etapas siguientes, entre las cuales se incluirá una triangulación de datos con las reacciones en otras plataformas al video focalizado, además de las entrevistas públicas ofrecidas por el exalcalde a propósito del hecho. Sobre ninguno de estos últimos datos se pretende dar cuenta en este artículo.

El resultado de las categorías emergentes y de las subcategorías focalizadas se sintetiza a continuación, como inventario de reacciones al video en YouTube. Posteriormente, se analizan los tipos de apoyo a la conducta del alcalde, las justificaciones desplegadas por los internautas a favor de la agresión física y, como conclusión, las reflexiones políticas que pueden derivarse.

\section{Resultados}

\section{Inventario de reacciones}

El inventario de reacciones fue favorable a la imagen pública del exalcalde de Bucaramanga. El ataque violento y explícito mostrado en el video fue apoyado por la mitad ( $52 \%$ ) de los internautas durante su primer mes de difusión, a través de comentarios que respaldaron la agresión, en diferentes grados y tonos. En otro grupo importante de comentarios (21\%), los internautas banalizaron la situación mofándose de ella o simplemente expresando risas y burlas. Un porcentaje minoritario manifestó su disgusto con la actitud del funcionario público y con la situación, avergonzándose de ella ( $11 \%)$ o bien reprobando explícitamente el ataque violento (9\%). Otros comentarios menos frecuentes se distribuyeron entre la incomprensión o la indiferencia frente al hecho ( $2 \%)$, apuntes heterogéneos de sorpresa, preguntas, reclamos, entre otros (3\%) e imputaciones o acusaciones contra el alcalde ( $1 \%)$ entre el 28 de noviembre y el 28 de diciembre de 2018 (Tabla 1).

Los resultados demuestran que las agresiones verbales y físicas pueden generar simpatías en entornos políticos mediatizados, especialmente cuando los protagonistas son figuras públicas expuestas a la evaluación perma- 
nente de sus conductas. La adhesión política por activación de emociones profundas parece tener más peso en estas situaciones que la objetivación de las agresiones como conductas inapropiadas para el debate argumentado. Esa adhesión se manifesta en cuatro formas de apoyo al agresor entre el 28 de noviembre y el 28 de diciembre de 2018, identificadas y ejemplificadas en la Tabla 2.

Tabla 1. Reacciones en YouTube a la agresión del alcalde

\begin{tabular}{|c|c|c|}
\hline \multirow{2}{*}{ Reacciones } & \multicolumn{2}{|c|}{ Frecuencia } \\
\cline { 2 - 3 } & abs. & rel. \\
\hline Apoyo & 253 & $52 \%$ \\
\hline Burla & 101 & $21 \%$ \\
\hline Vergüenza & 54 & $11 \%$ \\
\hline Reprobación & 46 & $9 \%$ \\
\hline Incomprensión/indiferencia & 11 & $2 \%$ \\
\hline Varios & 17 & $3 \%$ \\
\hline Imputación & 6 & $1 \%$ \\
\hline Total comentarios & 488 & $100 \%$ \\
\hline
\end{tabular}

Fuente: elaboración propia.

Tabla 2. Tipos de apoyo en YouTube a la agresión del alcalde

\begin{tabular}{|c|c|c|c|}
\hline \multirow{2}{*}{ Apoyos } & \multicolumn{2}{|c|}{ Frecuencia } & \multirow{2}{*}{ Ejemplos representativos } \\
\hline & abs. & rel. & \\
\hline Celebraciones & 104 & $41 \%$ & $\begin{array}{l}\text { "Este es el presidente que nos merecemos! Gracias ING Rodolfo } \\
\text { por ese golpe a la corrupción" }\end{array}$ \\
\hline Justificaciones & 95 & $38 \%$ & $\begin{array}{c}\text { "Pues si alguien llega a mi casa .. y luego me falta el respeto, se mete } \\
\text { con mi hijo.. yo le meto su arepaso pa que respete .. y que no se haga } \\
\text { la victima porque eso se llama provbocacion" }\end{array}$ \\
\hline Instigaciones & 33 & $13 \%$ & $\begin{array}{l}\text { "Lastima que no le partio la jeta a este concejal ladron, este alcalde } \\
\text { es de lo mejor" }\end{array}$ \\
\hline Aprobaciones & 21 & $8 \%$ & $\begin{array}{l}\text { "Soy de medellin y apoyo a esa berruga de alcalde, ese señor segun } \\
\text { lo que e leido de el a sido muy transparente en su mandato" }\end{array}$ \\
\hline Total apoyos & 253 & $100 \%$ & \\
\hline
\end{tabular}

Fuente: elaboración propia.

El 41 \% de los internautas de la muestra celebró la agresión física del alcalde con expresiones de felicitación y admiración o bien con censuras contra el agredido. Estas evaluaciones positivas aplaudieron el "carácter" del 
alcalde, su posicionamieto ideológico y su gestión contra prácticas corruptas, dentro de la cual se inscribiría la agresión contra el concejal.

Otra parte sustancial (38 \%) de los comentarios de apoyo trató de justificar la conducta del alcalde para suprimir la censura a la agresión física. Las justificaciones tienen especial interés por la verbalización de las excusas a favor de comportamientos irracionales por parte de las figuras políticas representativas. Los modos justificatorios se examinarán en el siguiente apartado.

El $13 \%$ de los internatuas llevó el apoyo hasta instigar actos aun más violentos contra el concejal o contra los siguientes sectores sociales focalizados: los concejales de Bucaramanga, la clase política corrupta y los simpatizantes de la izquierda ideológica. Finalmente, un porcentaje menor (8\%) de comentarios respaldó al alcalde sin legitimar su conducta agresiva, es decir, aprobó al mandatario, aunque no compartiera completamente su reacción violenta.

\section{Justificaciones de la agresión física}

El 38 \% de los comentarios digitales de apoyo justificó la agresión física del alcalde de Bucaramanga. Los resultados se distribuyen en tres tipos de justificación: provocación/entrampamiento; corrupción del enemigo y defensa intraespecífica, esta última dividida en dos subtipos: familiar y territorial (Tabla 3).

\section{Tabla 3. Tipos de justificaciones en YouTube a la agresión del Alcalde}

\begin{tabular}{|c|c|c|c|}
\hline \multirow{2}{*}{ Justificaciones } & \multicolumn{2}{|c|}{ Frecuencia } & \multicolumn{2}{c|}{ Ejemplos representativos } \\
\cline { 2 - 3 } & abs. & rel. & \\
\hline Defensa familiar & 25 & $26 \%$ & $\begin{array}{c}\text { "El alcalde tiene el derecho a enojarse así, osea como se va a meter } \\
\text { con su hijo" }\end{array}$ \\
\hline Defensa territorial & 24 & $25 \%$ & $\begin{array}{c}\text { "Bien rebien por el alcalde nadie puede ir ala casa de uno y tratarlo } \\
\text { mal ese lo quiero de presidente carajo" }\end{array}$ \\
\hline Corrupción del enemigo & 24 & $25 \%$ & "Así ay que darles a los consejales ratas asquerosas que se roban todo" \\
\hline $\begin{array}{c}\text { Provocación/ } \\
\text { entrampamiento }\end{array}$ & 22 & $23 \%$ & $\begin{array}{c}\text { "Lo hacen justamente para sacarlo de casillas y que reaccione así, a ver si por } \\
\text { ese lado si pueden quitarle el nombramiento como Alcalde. Pendejadas!!!" }\end{array}$ \\
\hline Total justificaciones & 95 & $100 \%$ & \\
\hline
\end{tabular}

Fuente: elaboración propia. 
Las justificaciones basadas en la defensa familiar (51\%) presentan los actos violentos ocurridos como agresiones reactivas naturales frente a la amenaza externa. Los internautas consideraron que la amenaza a la familia de alcalde y la intromisión en su casa por parte del concejal fueron las causas directas de la conducta violenta. En el primer caso, la base de la agresión justificada es su función de protección vital de los miembros más cercanos al agresor y, al mismo tiempo, de los más débiles dentro del círculo afectivo vinculante. En este sentido funcional de la protección del endogrupo (la familia del excalde agresor), la reacción agresiva contra las amenazas externas es una cuestión instintiva y pasional, ligada a la conservación intraespecífica e intensificada por la proximidad familiar de los seres defendidos (Lorenz, 2016, pp. 48, 53 y 212).

Los comentarios de los internautas no permiten concluir que ellos se agrupen, a su vez, en endogrupos o en el grupo familiar del exalcalde (esto requeriría un análisis de interacciones que en este artículo no se abordan), pero sí dejan ver que los foristas remarcan ese vínculo familiar del político explícitamente en la justificaciones que ofrecen³: "Si a mí me hablan mal de mi hijo también le meto la mano"; "El mejor alcalde de Colombia, aunque deberían mostrar el vídeo desde el principio, donde el concejal hace acusaciones contra el alcalde sin que él se pueda defender, finalmente se le mete con su hijo (con su familia) que es el punto débil de cualquier persona"; "Esos $\mathrm{HP}$ concejales no saben cómo joder a nuestro alcalde por eso se le metan con lo que más le duele a uno como padre que son los hijos muerte politica a todos esos bandidos que dicen representarnos en el concejo lo felicito alcalde mano fuerte para estirpar ese cáncer llamado concejo de bandidos”.

En los ejemplos se destacan las construcciones nominales que reelaboran formas empáticas de la justificación, a través de las cuales los internautas asumen como propia la acusación contra el hijo del alcalde y la convierten en una afrenta personal que excusaría la conducta violenta como reacción instintiva de protección a favor del más débil. Las justificaciones empáticas siguen el orden conductual de la repetición ("en su lugar, yo también...”), por identificación con el protector del endogrupo fami-

3 Todos las negrillas son añadidas a los originales. 
liar, y avanzan hacia la sanción del agredido físicamente, por atreverse a atacar a la familia del agresor: "Le dice Picaro al hijo del alcalde y que espera? Uno no se mete con la mama ni con los hijos de nadie, a menos que quiera insultar. Donde esta la sentencia concejal?"; "Ese concejal esta haciendo campaña desde hace rato y si a mi me habla mal de mi hijo sin pruebas tambien le meto la mano"; "Enrealidad la acusación del concejal no venia a cuento y fue solo para difamarlo frente a la camara, grave falacia; ademas se mete nada mas que con el hijo. Justa razon para darle ese pelmazo y cuidado que sino le doy en otra vez en la cara marica”.

El principio de la protección familiar (como remarca otro internauta usando la expresión popular "familia es familia") desacredita las acusaciones legales del concejal y las convierte en difamaciones censurables o en estrategias políticas sucias que justificarían la conducta violenta del alcalde. Por encima de cualquier sospecha o control político en el juego democrático, el imperativo de la defensa a toda costa de la familia particular del alcalde como su endogrupo borra la frontera entre las instituciones sociales y las reacciones viscerales.

La defensa del territorio privado, el hogar, forma parte de ese reemplazo de la discusión política por la justificación del ataque. La territorialidad aquí, como dimensión básica de la conducta humana (Eibl-Eibesfeldt, 2017, p. 339), otorga el derecho de regular la entrada de los individuos externos al sitio demarcado como propio y, en consecuencia, reclama la reacción violenta contra la amenaza externa en función de conservar el orden interno del grupo protegido. Así, el hecho de que el Alcalde esté dispuesto a defender su casa de la amenaza del concejal intruso parece excusar la agresión: "No comparto la agresion pero si mi insultas en mi casa tenga xa q Respete"; "obvio!.. el alcalde esta en su casa y hace respetar los derechos de su familia. el respeto, la paz y tranquilidad. en casa ajena se respeta"; "Se la gano bien ganada para que se iba a buscarlo a su casa...esos problemas se arreglan en otro lugar no en la casa del enemigo no ve que eso es desventaja”.

El énfasis en el lugar de los hechos es orientado por los internautas hacia el reclamo de "respeto"; es la transgresión del orden interno territo- 
rial del hogar lo que motiva la sanción contra el concejal agredido, presentado en los comentarios como el primer agresor que tendría ventaja sobre el enemigo al que invade en su propia casa. Sobre la base de la defensa territorial se construye la justificación que avanza hacia la evalación positiva del alcalde agresor: "Bien echo Sr Alcalde que tal ese H P llega a la casa a joderle la vida. No que tal y lo mata de un infarto. Asquerosos cpmo el alcalde no Roba como Ellos por eso lo desprestigian"; "Bien rebien por el alcalde nadie puede ir ala casa de uno y tratarlo mal ese lo quiero de presidente carajo".

Los comentarios digitales activan un contrato territorial implícito según el cual la invasión del espacio vital del otro tiene que esperar las peores consecuencias. Pero, además, son transgresiones que terminan favoreciendo al alcalde, porque provocan la solidaridad con él y lo proyectan como representante de las mayorías, el que está dispuesto a defender territorialmente su casa a cualquier precio. Esta forma de empatía con el agresor en el espacio público conlleva la demarcación del enemigo en clave de confrotación territorial: el invasor, en este caso, es sospechoso y censurable.

El $25 \%$ de las justificaciones acentúa esa representación negativa del agredido como un político corrupto. En este escenario, la bandera de la anticorrupción habilitaría la agresión física bajo la forma del castigo merecido: "Así es como se debe tratar a todas las ratas leguleyas de este país...concejal marullero ... irrespetuoso HP. Bravo alcalde ...lo necesitamos de presidente ....”

\footnotetext{
FELICITACIONES SEÑOR ALCALDE A LAS SABANDIJAS PONZOÑOSAS DE LOS CONCEJALES TOCA TRATARLOS ASI. Y EN LAS PROXIMAS ELECCIONES A BORRAR ESTA MANO DE LADRONES CHUPASANGRE A BASE DE COIMAS QUE EN ESTE PERIODO NO VIERON NI UN PESO POR ESO ESTAN ARDIDOS.PORQUE EL ALCALDE NO ES UN CORRUPTO COMO ELLOS. (mayúsculas en el original)
}

El discurso anticorrupción aquí reivindica la agresión como una respuesta necesaria, atravesada por la indigación popular frente al manejo indebido de las arcas públicas. La relación con la política electoral va a reforzar la justificación de la violencia, toda vez que es en el marco de la elección de los representantes donde se jugaría principalmente el castigo a los corruptos y el premio para los honestos. El castigo aquí ya no es simbólico ni democrático (abstenerse de elegirlos), sino directamente fáctico (golpearlos). Se- 
gún los internautas, el castigo físico es merecido además por la pertenencia del concejal agredido a la "mafia corrupta" que buscaría destituir al alcalde: "Estuvo bueno ese totaso mano de malparidos concejales de bucaramanga haciéndole la vida imposible al alcalde, como rodolfo si nos los a dejado robar a toda esa manada de hijueputas; "Ojo ojo bumangueses la mafia corrupta buscara que destituyan al alcalde, ni por el putas vayan a permitir esto este tipo lo necesitamos como presidente de Colombia cuidemoslo".

El despliegue de insultos acompaña regularmente la justificación de la agresión física para remarcar los sentimientos de indignación desbordada que movilizan la animadversión contra el agredido. Al inscribirlo en las formas nominales colectivas que se critican, la víctima de la agresión pasa a representar al enemigo como chivo expiatorio de la corrupción política. Asignar claramente ese rol resulta necesario en la lógica justificatoria de la conducta violenta.

Finalmente, el $23 \%$ de las justificaciones enfatiza que la reacción del alcalde fue causada por la provocación alevosa del concejal. Desde el punto de vista de estos internautas, el golpe es, simplemente, la respuesta natural e incotenible a un estímulo malintencionado o la respuesta lógica a un lugar común: "el que busca, encuentra": "Lo provoco a tal punto que se gano su manazo!!!"; "bien hecho; me alegra lo q el alcalde le hizo al concejal. el q busca encuentra”.

En el mismo sentido, toda la escena es definida como un entrampamiento que los internautas advierten y sancionan, sobre la base de la transparencia en el escenario político. Este principio conduce a evaluar negativamente la confrontación y los reclamos del concejal y, de ahí, a justificar el ataque del Alcalde: "La cámara y el micrófono ... un montaje descarado solo iba a provocarlo, que triste ver a Jhon Claro de bufón, muy bien por el alcalde, el cachetadón es el cierre perfecto para la payasada que le montaron, hasta dos le hubieran caído muy bien"; "Es cierto compañeros... Fue una trampa que le tendió este bandido con pinta de espantapájaros... Concejal tenía que ser... Vamos con toda con el Mejor... Oigan lo bien señores del Tiempo... Con el Mejor Alcalde de Colombia...”. 
Los comentarios digitales también reprocharon el tratamiento de la noticia en detrimento de la imagen del alcalde y defendieron una versión en su favor, en la cual la conducta impulsiva del funcionario público era excusable y comprensible, por haber sido provocada truculentamente por un personaje desacreditado. Los calificativos contra el concejal ("payaso", "bufón", "espantapájaros") orientaron su descalificación hacia la imposibilidad de tomar en serio las acusaciones, ya no solo por formar parte de un entrampamiento, sino, además, por provenir de una fuerza opositora sin capital político suficiente para hacerle contrapeso al poder gubernamental.

\section{Discusión}

En este artículo se propuso un análisis de los comentarios digitales publicados en el ciberforo de un video de noviembre de 2018, en el cual se registra el ataque físico y verbal del excalcalde de Bucaramanga contra un concejal de esa ciudad. Los resultados demuestran que esta conducta violenta produjo mínimos rechazos entre los internautas y, en cambio, obtuvo apoyo mayoritario a la figura del agresor.

La hipótesis planteada con base en las reflexiones de la etología humana sobre la agresión apuntó a interpretar las reacciones de los internautas de acuerdo con la lógica de las emociones básicas compartidas que pueden llevar a justificar conductas violentas. Los resultados demuestran que los espectadores movilizan la empatía con el agresor en función del mecanismo de protección del grupo familiar y en rechazo a las amenazas contra el territorio personal del político. Las justificaciones de la conducta agresiva confirman esa hipótesis y permiten seguir interrogando, en futuros trabajos, el funcionamiento específico en las plataformas digitales de aspectos filogenéticos de la conducta humana asociados a la agresión. La agresión del político fue apoyada con diferentes acentos. Las reacciones se inscribieron en un continuum cuyos extremos van desde la aprobación del candidato, a pesar de su conducta, hasta la instigación a ejecutar actos más violentos, como castigo merecido. En medio de esos extremos aparecen con mayor recurrencia comentarios que celebran la agresión del alcalde y otros que tratan de excusarla o minimizarla con justificaciones. 
La hipótesis, además, avanzaba hacia la explicación de las reacciones al video como una puesta en escena de emociones ciudadanas que transparentan tomas de postura en la política local, construida en gran medida por el tratamiento espectacularizante de las empresas informativas que se han visto obligadas a migrar o a hacer presencia en internet. En efecto, el video fue difundido en un canal de YouTube por parte de una de las casas de noticias de mayor tradición en Colombia (El Tiempo), sin ningún tipo de contextualización política, comentario editorial ni reflexión sobre sus implicaciones. Esa aparente neutralidad en la puesta a disposición del material en internet, como si se tratase de una escena sin contenido violento y que se bastara a sí misma, se apega a la lógica de los contenidos publicados en las redes sociales digitales, simplificados y de consumo directo, con propósitos más de entretenimiento que de información. Ese tratamiento de la noticia parece responder a la preferencia de los espectadores de YouTube por contenidos de esparcimiento y al decreciente interés por asuntos políticos de mayor complejidad (Rodríguez et al., 2018). En Gallardo y Alonso esa preferencia de los internautas ha sido explicada como una "traslación de las formas de consumo de la televisión tradicional” (2010, p. 434), que genera espectadores pasivos frente a los videos en YouTube.

La tendencia hacia el infoentretenimiento en internet, como lo discute Morini (2020), está haciendo que la cobertura mediática de las noticias políticas se centre en escándalos y controversias. Esa orientación va en detrimento de la función política y civil de los medios informativos (p. 108). Es claro que la producción de noticias también constituye un negocio con necesidades financieras dependientes del consumo y que, en las condiciones actuales del mercado de noticias en internet, la tentación de movilizar audiencias a través de encabezados emocionales sin contenidos sustanciales desafía el espíritu crítico y la responsabilidad de los medios informativos serios. Para Papacharissi (2018, p. 76), sin embargo, esos retos no impiden que el periodismo contemporáneo pueda utilizar los recursos de conexión y expresión de las plataformas digitales en beneficio de la convivencia y la cooperación. En el mismo sentido, Berry y Sobieraj (2014,p. 32) advierten que, aunque la difusión de discursos que promueven la agresión son rentables y eficaces para ganar apoyos políticos, socavan prácticas vitales para una vida democrática saludable. El caso de la agresión física presentada en 
YouTube como una noticia por parte de un medio informativo de renombre es una muestra de ese tipo de discursos que generan réditos económicos (para el medio) y políticos (para el exfuncionario público), pero poca reflexión sobre los asuntos de fondo.

El análisis de las reacciones a esa "noticia" mostró que los modos de la justificación se asentaron en condicionamientos básicos de la conducta agresiva. Los internautas hicieron énfasis en esos estímulos que instintivamente activarían la agresión para neutralizar las amenazas externas. Con ello, el ataque del alcalde resultó naturalizado y se obturaron las denuncias que el concejal pretendía difundir en la escena pública. Los aportes de los estudios etológicos ayudan a explicar, en clave sociobiológica, la base de esas justificaciones como: 1 ) defensas intragrupales, tanto de la familia como del territorio, para proteger el orden vital del endogrupo;2) respuestas proporcionales a una agresión provocadora y alevosa; y 3 ) reacciones necesarias frente a la capacidad de daño del enemigo inmoral, en particular, acusado de corrupción.

El marco etológico contemporáneo (Hammerstein, 2013; Kortüm y Heinze, 2013) no propone ningún determinismo conductual, sino que deshace la vieja dicotomía entre causas naturales y culturales para buscar explicaciones más integrales de las reacciones humanas en sus ámbitos específicos. En ese sentido, el análisis de la agresión no puede ignorar su herencia vital, la misma que predispone al hombre naturalmente tanto para la lucha como para la cooperación (De Waal, 2007). En el caso analizado, las condiciones del escenario público de la política, espectacularizado por los medios informativos en internet, predisponen a la lucha, desbordan los inhibidores de la agresión física y activan la empatía con esa conducta acudiendo a sus bases más instintivas: la defensa familiar/territorial y el mantenimiento de la jerarquía instituida.

\section{Conclusiones}

En este artículo se ha optado por una perspectiva interdisciplinar del Análisis del Discurso para abordar las reacciones a un caso de agresión física transmitida en video y comentada en una red social. Para interpretar la fa- 
vorabilidad de esas reacciones frente a la conducta agresiva del funcionario público que protagoniza la escena, se buscó dialogar con el enfoque que proporciona la etología humana sobre la agresión y, particularmente, sobre la justificación del ataque físico como defensa frente a amenazas intraespecíficas. Dado que no existen estudios ampliamente visibles que establezcan esta relación entre etología y comunicación política en plataformas digitales, este artículo propone iniciar reflexiones en ese sentido desde los contextos de la política local; por la misma razón, tiene carácter seminal y exploratorio. Es claro que este avance es apenas un punto de partida para actualizar o revisar las nociones etológicas clásicas en el marco de la conducta humana en redes sociales virtuales, ámbito estimulante para realizar abordajes interdisciplinares como el que se propone en este artículo, pero que no es posible agotar ni profundizar en un solo texto.

El alcance del artículo ha sido demostrar que los comentarios en YouTube son un escenario interesante para visualizar reacciones justificatorias frente a conductas agresivas de protagonistas políticos. Esas reacciones digitales constituyen una válvula de escape de las emociones ciudadanas y ponen en escena sus "tomas de postura" casi sin filtros. No obstante, su capacidad de predecir preferencias políticas reales es limitada y no debería conducir a conclusiones apresuradas sobre las matrices ideológicas imperantes ni sobre las elecciones futuras. Asimismo, el artículo se concentró en las reacciones, mas no en las interacciones entre internautas y, por tanto, el aporte del estudio acerca de los posibles agrupamientos ideológicos y construcción de polémicas queda pendiente para una segunda fase de investigación. La contribución de este análisis se encuentra más en el sentido de las reflexiones que habilita en torno a la democracia representativa y a la aparición de imposturas antipolíticas en el discurso y en la acción pública de quienes gobiernan, que efectivamente logran ganar simpatías en algunos sectores de la población.

La empatía con el político agresor reemplaza la promesa de la representación democrática encarnada en el más apto por la urgencia de ser representados por el más fuerte, esto es, aquel que garantice a cualquier costo la protección contra las amenazas externas. Lo que para Rosanvallon (2007) es un síntoma de la desconfianza en el sistema mismo de representación, 
como reacción a sus disfuncionalidades originales, puede comprenderse también sociobiológicamente como la activación de los mecanismos naturales agresivos que forman parte de las conductas humanas básicas y que ejercen influencia aun en el seno de sus instituciones culturales más sofisticadas, como puede serlo la democracia representativa.

En términos pasionales, nos identificamos más fácilmente con esas conductas naturales que con los mecanismos inhibidores de ellas, específicamente con los esfuerzos de hacer de la política un campo de discusiones razonadas y reguladas en las cuales la agresión física es sancionada. La masiva participación en el ciberforo analizado y su apoyo al alcalde agresor dan prueba de esa facilidad para empatizar con las conductas violentas, desde la tribuna del anonimato. Sin embargo, como vimos, en el ámbito de lo público la defensa de la agresión no siempre es directa y puede aparecer justificada para favorecer la imagen del político y su proyección en el poder gubernamental. En estos casos, lo que se presenta como reacción natural, visceral e inevitable contribuye a la formulación de una impostura antipolítica, esto es, a la imagen del sujeto que, por auténtico, se permite renunciar al hacer, al decir y al ser "políticamente correcto", con lo cual se le atribuye el derecho a transgredir las normas del ejercicio representativo en la democracia, pues, finalmente, es un ejercicio anclado en un sistema en el que ya pocos confían. Así, el precio más alto de la desconfianza en el sistema representativo y de la desafección política es el aplauso a los violentos, la celebración de las agresiones en las interacciones públicas y la preferencia por los más irreflexivos.

\section{Agradecimientos}

El autor expresa su agradecimiento a los evaluadores anónimos de este artículo, que promovieron la cualificación del texto a través de su juiciosa valoración y sus sugerencias bibliográficas.

\section{Referencias}

Abad, A. y Trak, J. M. (2013). Desafección política en Bolivia, Ecuador y Venezuela en 2010: un análisis comparado. Cuadernos del Cen- 
des, 30(82), 35-66. http://ve.scielo.org/scielo.php?script=sci arttext\&pid=S1012-25082013000100004\&lng=es\&tlng=es

Alloca, K. (2018). Videoycracy. How YouTube is changing the world. Nueva York: Bloomsbury.

Almond, G. (1990). The study of political culture. En G. Almond, A discipline divided. Schools and Sects in Political Science (pp. 138-156). Newbury Park: Sage.

Amossy, R. (2014). Apologie de la polémique. París: PUF. https://doi. org/10.3917/puf.amos.2014.01

Arnoux, E. (2019). El análisis del discurso como campo académico y práctica interpretativa. En Ó. I. Londoño y G. Olave (coords.), Métodos de análisis del discurso. Perspectivas argentinas (pp. 19-40). Bogotá: Ediciones de la U.

Bañuelos, J. (2009). YouTube como plataforma de la sociedad del espectáculo. Razón y Palabra, 66, 1-29. http://www.ww.w.razonypalabra. org.mx/N/n66/varia/jbanuelos.pdf

Barton, D. y Lee C. (2013). Language online. Investigating digital texts and practices. Londres-Nueva York: Routledge.

Berry, J. M. y Sobieraj, S. (2014). The outrage industry: Political opinion media and the new incivility. Oxford: Oxford University Press.

Boczkowski, P. y Papacharissi, Z. (eds.) (2018). Trump and the Media. The MIT Press. https://doi.org/10.7551/mitpress/11464.001.0001

Briceño, Y., Acevedo, M., Cogollo, D. y Sanabria, L. (2017). Interacción ciudadana frente al mensaje de un actor político emergente en Facebook. Revista La Tercera Orilla, 19, 42-56. https://doi. org/10.29375/21457190.2889 
Briceño, Y., Manrique, J., Sanabria, L. y Gómez, A. (2018). Clasificación discursiva de los comentarios en Facebook desde la actitud política. Index.comunicación, 8(3), 43-64. https://journals.sfu.ca/indexcomunicacion/index.php/indexcomunicacion/article/view/425

Burgess, J. y Green, J. (2009). YouTube. Digital Media and Society Series. Cambridge: Polity Books.

Di Palma, G. (1970). Apathy and Participation. Mass Politics in Western Societies. Nueva York: The Free Press.

De Waal, F. (2007). El mono que llevamos dentro. Barcelona: Tusquets.

Du Bois, J. W. (2007). The stance triangle. En R. Englebreston (ed.), Stancetaking in discourse: Subjectivity, evaluation, interaction (pp.39-182). Amsterdam: John Benjamins.

Dyke, S. (2013). Utilising a blended ethnographic approach to explore the online and offline lives of pro-ana community members. Etnografía y Educación, 8(2), 146-161. https://doi.org/10.1080/174578 23.2013.792505

Eibl-Eibesfeldt, I. (2017). Human Etology. Routledge. https://doi. org/10.4324/9780203789544

Eisenhardt, K. (1989). Building theories from case study research. Academy of Management Review, 14(4), 532-550.https://doi.org/10.5465/ amr.1989.4308385

Englebretson, R. (2007). Stancetaking in Discourse. Subjectivity, evaluation, interaction. Londres: John Benjamins. https://doi.org/10.1075/ pbns. 164

Forni, P. (2011). Los estudios de caso: orígenes, cuestiones de diseño y sus aportes a la teoría social. Miríada. Investigación en Ciencias Socia- 
les, 3(5), 61-80. https://p3.usal.edu.ar/index.php/miriada/article/view/5/153

Fracchiolla, B. (2013). De l'agression à la violence verbal, de l'éthologie à l'anthropologie de la communication. En B. Fracchiolla, C. Moïse, C. Romain y N. Auger (eds.), Violences verbales: Analyses, enjeux et perspectives (pp. 19-36). Rennes: Presses Universitaires de Rennes.

Gallardo-Camacho, J. y Alonso, J. (2010). La baja interacción del espectador de vídeos en Internet: caso Youtube España. Revista Latina de Comunicación Social, 65, 421-435. https://doi.org/10.4185/ RLCS-65-2010-910-421-435

Graf, J., Erba, J. y Harn, R. W. (2017). The role of civility and anonymity on perceptions of online comments. Mass Communication and Society, 20(4), 526-549. https://doi.org/10.1080/15205436.2016. 1274763

Hammerstein, P. (2013). What theoretical biology has to say on aggression in humans and animals. En H. Kortüm y J. Heinze (eds.), Aggression in humans and other primates (pp. 23-40). Berlín: Degruyter.

Happer, C., Hoskins, A. y Merrin, A. (eds.) (2019). Trump's Media War. Suiza: Palgrave MacMillan. https://doi.org/10.1007/978-3-31994069-4

Hernández, R., Méndez, S., Mendoza, C. y Cuevas, A. (2017). Fundamentos de la investigación. México: McGraw-Hill.

Jaffe, A. (ed.) (2009). Stance. Sociolinguistic perspectives. Oxford: Oxford University Press. https://doi.org/10.1093/acprof:o so/9780195331646.001.0001

Klostermann, P. (2015). YouTube Comment Scraper. http://ytcomments. klostermann.ca/ 
Kortüm, H. y Heinze, J. (eds.) (2013). Aggression in humans and other primates. Berlín: Degruyter.

Ksiazek, T., Peer, L. y Zivic, A. (2015). Discussing the news: Civility and hostility in user comments. Digital Journalism, 3(6), 850-870. https://doi.org/10.1080/21670811.2014.972079

Kwon, K. y Gruzd, A. (2017). Is aggression contagious online? A case of swearing on Donald Trump's campaign videos on YouTube [Proceedings]. $50^{\text {th }}$ International Conference on System Sciences, Hawaii. https:// doi.org/10.24251/HICSS.2017.262

Lange, P. (2014). Commenting on YouTube rants: Perceptions of inappopriateness or civic engagement? Journal of Pragmatics, 73, 53-65. DOI: https://doi.org/10.1016/j.pragma.2014.07.004

Lanza, L. y Fidel, N. (2011). Política 2.0 y la comunicación en tiempos modernos. Centro de Estudios en Diseño y Comunicación, 35, 53-63. https://doi.org/10.18682/cdc.vi35

Lorenz, K. (2016). Sobre la agresión. El pretendido mal. F. Blanco (trad.). México: Siglo XXI.

May, A. (2010). Who Tube? How YouTube's news and politics space is going mainstream. The International Journal of Press/Politics, 15 (4), 499511. https://doi.org/10.1177/1940161210382861

McCosker, A. (2013). Trolling as provocation: YouTube's agonistic publics. Convergence 20(2), 201-217. https://doi. org/10.1177/1354856513501413

Moor, P., Heuvelman, A. y Verleur, R. (2010). Flaming on YouTube. Computers in Human Behavior, 26, 1536-1546. https://doi.org/10.1016/j. chb.2010.05.023 
Morini, M. (2020). Lessons from Trump's political communication. How to dominate the media environment. Suiza: Palgrave MacMillan. https:// doi.org/10.1007/978-3-030-39010-5

Mouffe, Ch. (2003). La paradoja democrática. Barcelona: Gedisa.

Novaro, M. (2000). Representación y liderazgo en las democracias contemporáneas. Buenos Aires: Homo Sapiens.

Olave, G. (2019). Agresiones verbales en streaming: la disputa pública por la implementación del Acuerdo de Paz en Colombia [Ponencia], I Congreso Iberoamericano de Argumentación, agosto 14-16, Universidad EAFIT, Medellín.

Papacharissi, Z. (2018). The importance of being a headline. En P. Boczkowski y Z. Papacharissi (eds.), Trump and the Media (pp. 7178). Cambridge, Mass: The MIT Press.

Pardo, N. (2012). Discurso en la Web: pobreza en YouTube. Bogotá: Universidad Nacional de Colombia.

Reddy, W. (2001). The navigation of feeling. A framework for the history of emotions. Cambridge: Cambridge University Press.

Rodríguez-Breijo, V., Gallardo-Camacho, J.y Sierra-Sánchez, J. (2018). Información política en los vídeos que son tendencia en YouTube España. El profesional de la información, 27(5), 1041-1049. https:// doi.org/10.3145/epi.2018.sep.08

Rosanvallon, P. (2007). La contrademocracia. La política en la era de la desconfianza. Buenos Aires: Manantial.

Salcedo, L. y García, J. (2015). La desafección política en Colombia: un análisis sistémico al respecto. Económicas CUC, 36(2), 49-65. https:// doi.org/10.17981/econcuc.36.2.2015.4 
Schmitt, A., Atzwanger, K., Grammer, K. y Schäfer, K. (eds.) (1997). New aspects of human ethology. Nueva York/Londres: Plenum Press. https://doi.org/10.1007/b102412

Schneebeli, C. (2015). Les modalités linguistiques du commentaire sur internet comme prise de position ("stance-taking"): l'exemple des commentaires sur YouTube. HAL-Sciences del Homme et de la Société. https://halshs.archives-ouvertes.fr/halshs-01406715/

Scolari, C. (2004). Hacer clic. Hacia una sociosemiótica de las interacciones digitales. Madrid: Gedisa.

Staiger, J., Cvetkovich, A. y Reynolds, A. (2010). Political emotions. New agendas in communication. Nueva York: Routledge. https://doi. org/10.4324/9780203849538

Stake, R. (1995). Investigación con estudios de caso. Madrid: Morata.

Strauss, A. y Corbin, J. (2002). Bases de la investigación cualitativa. Técnicas y procedimentos para desarrollar la teoría fundamentada. Medellín: Universidad de Antioquia.

Wahl-Jorgensen, K. (2018). Public displays of disaffection: The emotional politics of Donald Trump. En P. Boczkowski y Z. Papacharissi (eds.), Trump and the Media (pp. 79-86). Cambridge, Mass: The MIT Press.

Yin, R. (1984). Case study research. Design and methods. Thousand Oaks: Sage. 